\title{
ECOLOGIA DELLA PROTESTA. COME IL DIRITTO OSSERVA LA DISOBBEDIENZA
}

\author{
Corrado Punzi \\ Università del Salento
}

https://doi.org/10.33676/EMUI_nomads.54.02

\begin{abstract}
IT).- II contributo descrive la teoria dei sistemi come una teoria ecologica dei movimenti ecologisti, capace di radicalizzare il rapporto tra sistema e ambiente e di superare i limiti epistemologici di ogni prospettiva antropocentrica e appropriativa della natura. Il movimento No Tap, costituitosi nel Salento per opporsi alla costruzione di un gasdotto finanziato dall'Unione Europea, viene osservato come un caso paradigmatico delle forme moderne di disciplinamento giuridico-amministrativo del conflitto, delle tecniche di immunizzazione della protesta. Verrà quindi affrontata la questione arendtiana del rapporto tra disobbedienza e diritto, interrogandosi sulla funzione dei procedimenti giuridici nei confronti della protesta e su come il diritto possa o meno trattare il rischio, cioè il non-sapere del futuro.
\end{abstract}

\section{Ecology of protest. How law observes disobedience}

Abstract (EN).- The contribution describes the theory of systems as an ecological theory of ecological movements, capable of radicalizing the relationship between the system and the environment and overcoming the epistemological limits of every anthropocentric and appropriative perspective of nature. The No Tap movement, established in Salento to oppose the construction of a gas pipeline financed by the European Union, is seen as a paradigmatic case of the modern forms of legal and administrative regulation of the conflict, of the immunization techniques of the protest. The Arendt's question of the relationship between disobedience and law will then be addressed, questioning the function of legal proceedings against the protest and how the legal system can treat the risk, that is the ignorance of the future.

Keywords: movimenti di protesta, rischio, processi partecipativi, procedimenti giuridici, protest movements, risk, partecipatory processes, legal proceedings

\section{Osservare la società del rischio e della protesta}

Nel 1990 Niklas Luhmann e Raffaele De Giorgi fondarono a Lecce il Centro di Studi sul Rischio, nella convinzione che la società moderna fosse (già) la società del rischio (e del pericolo). Una società senza referenze esterne di orientamento e quindi abbandonata a se stessa, al suo decidere in condizioni d'incertezza, cioè di nonsapere del futuro (Luhmann 2006a). Una società che in ogni decisione che prende (o che non-prende) produce possibili danni ovvero rischi per i decisori e simultaneamente pericoli per i coinvolti, cioè per la maggioranza. Una società, quindi, 
che produce anche continuamente situazioni affini alla protesta, perché si confronta sempre con il problema della minaccia e del futuro che non si realizza come ci si attende (Corsi 2003: 285). Perciò la società del rischio è anche la società della protesta o la società di movimento (Meyer and Tarrow 1998).

Si potrebbe pensare che la nascita del Centro di Studi sul Rischio a Lecce potesse essere connessa a una particolare attenzione territoriale al problema del rischio 0 , al limite, che la potesse favorire. Ma non è così. E non lo è nonostante nel 1990, a pochi chilometri da Lecce, fossero già attive da decenni alcune tra le più imponenti industrie europee: l'acciaieria Ilva di Taranto e il Petrolchimico Eni di Brindisi. E nonostante a Cerano, ad appena 33 chilometri da Lecce, fosse appena stata costruita la Centrale a carbone Enel. In modo del tutto sorprendente e paradossale, nel territorio salentino il tema del rischio da inquinamento da energie fossili aveva una risonanza quasi nulla. La comunicazione, infatti, era (ancora) canalizzata sulle possibilità di sviluppo del territorio, individuate paradossalmente tanto nella creazione di piani industriali e di lavoro, quanto nella promozione di un turismo alla ricerca di paesaggi incontaminati. D'altra parte, si riteneva che proprio la natura (e la vita) incontaminata costituisse(ro) il tratto distintivo della propria identità meridionale, cioè della propria differenza con il nord. Nell'ultimo decennio, tuttavia, questa autodescrizione è implosa su se stessa, davanti alle evidenze scientifiche dei primi studi epidemiologici che hanno stabilito un nesso tra le emissioni delle industrie fossili e percentuali di tumori molto più elevate della media europea. II territorio ha così iniziato lentamente a tematizzare la connessione tra il rischio industriale e la malattia, tanto dei sistemi organici quanto di quelli psichici. Tuttavia, i decisori politici ed economici hanno continuato a mantenere ferme le proprie costruzioni semantiche del territorio e le proprie aspettative; di conseguenza, davanti a questa ostinata persistenza, è sembrato che i coinvolti sparissero dalla sfera pubblica e si chiudessero privatamente in una Spirale del silenzio (Noelle-Neumman 2002) e della vergogna: per le proprie precedenti autodescrizioni, per la credulità manifestata nei confronti delle retoriche sviluppiste e, perfino, per la colpa della propria malattia. A rompere parzialmente questa spirale del silenzio, ha contribuito dal 2011 l'intenzione europea e italiana di approvare il progetto di costruzione di un mega-gasdotto di $4000 \mathrm{~km}$, per il trasporto di gas dall'Azerbaijan all'Europa. Contro l'approdo sulla costa della provincia di Lecce, si è costituito il movimento No Tap. Dopo anni di protesta e di conflitto giuridico-amministrativo, il progetto è stato però definitivamente approvato e avviato nel 2017. Tuttavia, nonostante i sigilli istituzionali, il movimento di opposizione a Tap si è progressivamente ampliato, congiuntamente alle forme del conflitto e del disciplinamento giuridico della protesta. D'altra parte, in questi anni, l'autodescrizione del territorio è radicalmente mutata: i movimenti ecologisti, infatti, hanno contribuito a fornire risonanza al tema del pericolo industriale, agitando lo spettro della paura e trasformando l'incertezza del rischio in certezza del danno e il non-sapere del futuro nel sapere dell'attesa tragica di una distopia.

Questo contributo non intende analizzare il movimento No Tap, ma tramite esso provare a descrivere e a utilizzare la teoria dei sistemi sociali di Niklas Luhmann (1984) come una teoria ecologica dei movimenti ecologisti. Riferirsi al movimento di protesta 
No Tap ${ }^{1}$ può essere utile, pertanto, per verificare le potenzialità empirico-esplicative di una teoria costruita con pretese di astrazione. Risulta interessante comprendere se la teoria dei sistemi possa rispondere alle principali questioni sociali e filosofico-giuridiche sollevate dal tema e poste già da Hannah Arendt (2017) nella sua riflessione sulla Disobbedienza civile. Qual è la compatibilità tra disobbedienza civile e legge? Qual è la legittimità del contratto sociale, ossia il rapporto tra consenso tacito e dissenso esplicito? E di conseguenza: qual è la capacità della democrazia di rapportarsi con le minoranze disobbedienti e con il desiderio di cambiamento dei movimenti? In sintesi: con quale concezione di diritto è compatibile la protesta (ecologista)?

Per tentare di rispondere a queste domande è necessario, però, fare alcune precisazioni sulla teoria dei sistemi. Spesso, infatti, si è ritenuto che essa non solo non si occupasse di analizzare i problemi concreti della società, ma che fosse una teoria conservatrice, per nulla interessata al tema del cambiamento sociale e quindi della disobbedienza e dei movimenti. Al contrario, già dagli anni Ottanta Luhmann ha affrontato il tema dei nuovi movimenti sociali e della protesta (Luhmann 1986), delle minacce ecologiche e del rischio ambientale (Luhmann 1989), come riflessioni propedeutiche per la futura costruzione di una Sociologia del rischio (Luhmann 1996). I movimenti di protesta sono stati osservati come nuovi movimenti sociali, nati dalla fine degli anni Sessanta, quando in Europa si diffondono i movimenti studenteschi e poi quelli femministi, pacifisti ed ecologisti2. Secondo il sociologo e filosofo tedesco, la novità dei nuovi movimenti sociali non sta in un'ideologia innovativa o in "resti sparpagliati di pretese di legalità e di solidarietà economica; essa sta in un nuovo tipo di protesta: nel rifiuto di situazioni nelle quali si potrebbe diventare la vittima del comportamento rischioso di altri" (Luhmann 1996: 155). I nuovi movimenti sociali, quindi, si orientano sempre meno ai disordini interni, cioè alle diseguaglianze sistemiche, e sempre più ai disordini esterni, cioè al disequilibrio ambientale o ecologico (Luhmann 2006b: 672-686). Essi proliferano sulle angosce generate dal nonsapere del futuro e il conflitto che vivono è proprio sull'interpretazione del non-sapere: si sentano spettatori di una (auto-) distruzione annunciata e protestano contro i decisori.

La seconda precisazione necessaria riguarda le accuse pregiudiziali di conservatorismo avanzate nei confronti della teoria dei sistemi. Al contrario di quanto pensino i suoi detrattori, essa non si occupa della stabilità delle strutture sociali, ma piuttosto della loro evoluzione, descritta come una improbabilità che si rende

\footnotetext{
1 Negli ultimi tre anni ho seguito e osservato le attività del Movimento No Tap, realizzando anche una serie di interviste in profondità ai suoi principali attivisti. Ho poi osservato delle giornate di democrazia partecipativa - organizzate dalla Regione Puglia - e diverse manifestazioni e incontri pubblici organizzati dal movimento. Ho inoltre analizzato alcuni atti e provvedimenti della Questura e della Prefettura di Lecce contro il movimento e, infine, ho osservato come vecchi e nuovi media hanno interpretato la vicenda e, in particolare, come Tap e movimento di protesta abbiano costruito la propria immagine e cercato risonanza mediatica. Da questa ricerca empirica, nascono anche le presenti riflessioni teoriche.

2 Come evidenzia Pieroni (2002: 240), è stato soprattutto il movimento femminista, centrato sull'etica della cura e della relazione, ad anticipare le poste in gioco dei conflitti della società contemporanea e del movimento ecologista.
} 
probabile da sé3. La prospettiva sistemica, infatti, rifiuta qualsiasi rappresentazione normativo-escatologica della realtà, che evidentemente deriva dal volersi collocare nella posizione di chi sa meglio degli altri in che direzione la società stia andando o debba andare. Luhmann (2017: 66), invece, è interessato a dirci soltanto dove la società è. Per questo, coloro che credono che la teoria dei sistemi sia conservatrice potrebbero domandarsi se a essere conservatrice non sia piuttosto la realtà: politica, economica, giuridica e così via. A partire da questo presupposto teorico, il problema non è più se la teoria dei sistemi possa rispondere alle questioni poste da Hannah Arendt, ma piuttosto come le osservi e, di conseguenza, come risponda. Risulta ora evidente, infatti, come l'approccio sistemico non indichi e non possa indicare quale concezione di diritto sia compatibile con la protesta (ecologista), né tantomeno possa proporre, come fá Arendt (2017: 60), di costituzionalizzare la disobbedienza civile. Luhmann, infatfi, costruisce una teoria che osserva come la realtà risponda fattualmente alle domande poste, aiutando a descrivere quale sia la concezione del diritto (e della politica e dell'economia) vigente e, poi al limite, lascia comprendere se essa sia o meno compatibile con la protesta.

Pertanto, attraverso la cornice della teoria dei sistemi si proverà a rispondere a queste nuove domande, nel tentativo di dimostrare come l'osservazione del Movimento No Tap possa costituire un caso paradigmatico delle forme moderne di disciplinamento giuridico del conflitto e, in particolare, di uno dei suoi due lati: quello della protesta. Al crescere della protesta, infatti, è avvenuta una "dislocazione del problema" 4 all'interno dei confini giuridici e politico-amministrativi. Così, seppur in ambiti differenti, il conflitto è stato progressivamente giuridicizzato, in quanto anche l'amministrazione è ricorsa all'uso di procedimenti giuridici, definiti tali non perché operanti esclusivamente nel sistema giuridico, ma in quanto "giuridicamente regolati", nel senso di finalizzati alla produzione di decisioni vincolanti e legittime (Luhmann 1995). Tale giuridicizzazione è avvenuta in due fasi principali. La prima fase ha coinvolto sia la multinazionale Tap sia il Governo, entrambi interessati a legittimare giuridicamente le loro pretese tramite il rispetto dei procedimenti amministrativi (Luhmann 1995): tanto delle procedure autorizzative (sia statali sia europee), quanto dei procedimenti di democratizzazione, cioè di coinvolgimento popolare per la deliberazione finale. La seconda fase, invece, è consistita nell'utilizzo di procedimenti specificamente giuridici: dapprima, gli attori sociali coinvolti sono ricorsi a tutte le possibili procedure giuridiche

\footnotetext{
3 Luhmann, infatti, pur ispirandosi allo struttural-funzionalismo di Parsons, lo rivisita radicalmente, trasformandolo in funzional-strutturalismo, cioè in una teoria che non è interessata tanto al modo in cui le strutture sociali possano ottenere e garantire la propria stabilità, quanto piuttosto alle funzioni e al loro senso in determinate situazioni: le strutture sociali non sono più considerate, insomma, come "dati ultimativi". D'altra parte, le critiche nei suoi confronti «semplicemente scambiano per difetti e carenze ciò che, nell'ottica di Luhmann, è invece il risultato di una consapevole scelta di diversificazione operata non solo nei contenuti ma anche delle pretese esplicative di altre teorien. (Febbrajo 1975: 30 e 38)

4 Luhmann (1974) parla di "dislocazione del problema" per indicare una delle strategie sociali utilizzate per tradurre la estrema complessità dell'ambiente in complessità interna, "che permette di identificare il problema come problema del sistema, rendendolo così risolvibile entro l'arco di possibilità delimitato dal sistema stesso" (De Giorgi 1998: 225).
} 
per risolvere il conflitto, paradossalmente accentuato dai procedimenti amministrativi; successivamente, dopo che il diritto ha deliberato in favore della multinazionale e del Governo, il movimento No Tap ha reagito alla delusione delle proprie aspettative giuridiche optando per l'organizzazione di presidi e manifestazioni non violente nei pressi del cantiere del gasdotto; così, davanti alla persistenza della protesta e alla sua de-istituzionalizzazione, il sistema giuridico non ha agito più soltanto come arbitro, ma è intervenuto attivamente tramite procedimenti giuridici d'eccezione, consistenti nell'esibizione del monopolio della forza fisica e nell'adozione di misure giuridicoamministrative repressive, come gli arresti e i fogli di via. Descrivere brevemente queste tappe della giuridicizzazione del conflitto significa anche rispondere sinteticamente alle precedenti questioni teoriche sul rapporto tra disobbedienza e diritto, traducendole operativamente nelle domande sulla funzione dei processi partecipativi neí confronti della protesta, sul rapporto tra diritto e non-sapere, sulla forza di legge, sulla funzione (evolutiva) della protesta e, infine, sul potenziale ecologicamente esplicativo della teoria dei sistemi.

\section{II disciplinamento giuridico del conflitto}

Sempre più, nella realizzazione delle grandi opere, gli organi amministrativi ricorrono all'utilizzo di procedimenti di democratizzazione delle decisioni: i cosiddetti processi partecipativi (dal basso), che mirano al coinvolgimento popolare nei processi decisionali preliminari alla costruzione. Questi tentativi, tuttavia, più che l'inizio di un coinvolgimento dei territori segnano quasi sempre l'avvio di una progressiva procedimentalizzazione del conflitto. È bene ricordare, infatti, ciò che quasi mai ricorda chi organizza questi processi partecipativi: ossia che le direttive europee impongono che l'approvazione della VIA (Valutazione di impatto ambientale), cioè una delle principali autorizzazioni a costruire, sia condizionata dalla attuazione di procedure che consentano di vagliare il parere delle comunità e delle amministrazioni locali, al fine di ottenere una "leale collaborazione tra istituzioni". Pertanto, il coinvolgimento dei territori non è partecipato, come si vorrebbe far credere, ma esclusivamente procedimentale: la sua funzione non è favorire una gestione orizzontale del problema, quanto piuttosto rispettare le procedure giuridicoamministrative imposte dall'Europa. Tuttavia, le normative europee non definiscono delle modalità di attuazione, ma impongono semplicemente degli obiettivi; allo stesso modo i procedimenti, per forma e per struttura, non stabiliscono "una rigida successioni di determinate azioni" (Luhmann 1995: 31). Così, agli organi amministrativi viene lasciata piena libertà interpretativa e attuativa, che nel caso Tap si è tradotta in un rispetto formale ma non sostanziale dei "vincoli" europei. Pertanto, la prima tappa di democratizzazione delle decisioni - anche nel caso del movimento No Tap -, più che l'inizio di un coinvolgimento dei territori, segna l'avvio di un primo tentativo di disciplinamento del dissenso. D'altra parte, come sostiene Luhmann, la funzione dei procedimenti partecipativi è legittimare il potere dei coinvolti, ma soltanto allo scopo di imbrigliarlo. Attraverso l'uso di parole d'ordine quali partecipazione e cogestione si suggerisce ai coinvolti la falsa coscienza che essi abbiano potere: 
[C]osì l'emancipazione diviene l'ultimo trucco del management: negando la differenza tra i superiori ed i subordinati si privano i subordinati della loro base di potere. Mentre si finge di livellare il potere, non si fa altro, in realtà, che riorganizzare quella parte del potere che tutto sommato è già nelle mani dei subordinati (Luhmann 1979: 129).

In questo modo il potere viene trasformato in potere sotto controllo, in un potere chiuso (Canetti 2004)5. L'espediente della democratizzazione del potere consisterebbe, quindi, nel livellare it potere al fine di riconoscere ai "subordinati" un potere formale (fittizio) e di privarli del loro incontrollato potere potenziale (sostanziale). Così la protesta potrebbe essere canalizzata in forme di disobbedienza civile e disciplinata, e non in forme che costringono all'uso della forza fisica, che tra l'altro richiederebbero di sostenere costi economici e politici; infatti, quando un potere è costretto a ricorrere alla violenza manifesta tutta la sua debolezza: si è molto più forti quando si controlla senza esercitare alcun controllo (Luhmann 1979). D'altra parte l'esercizio della forza comporta un elevato rischio di perdita del consenso, che deve essere controbilanciato da investimenti nella comunicazione per screditare la protesta e collocarla in uno spazio extra-giuridico. Pertanto, comprendere "I'ultimo trucco del management" significa comprendere che le procedure di partecipazione sono solo una finzione giuridico-amministrativa, una nuova economia del potere, molto più conveniente e meno rischiosa, ma la cui funzione latente è equivalente alla funzione esercitata dall'utilizzo della forza fisica: rendere immune alla protesta il sistema economico e, simultaneamente, quello politico e giuridico.

Se un movimento di protesta resiste ai primi tentativi di istituzionalizzazione e disciplinamento attuati con i processi partecipativi, si trova davanti all'alternativa di alzare il livello del conflitto da comunicativo a fisico o di utilizzare tutti i possibili procedimenti giuridici per ostacolare il progetto. Così, ad esempio, il movimento No Tap ha chiamato in causa il diritto, attivando procedimenti giuridici affinché osservassero la legittimità dei procedimenti amministrativi. In questo modo, un movimento può tematizzare la propria protesta all'interno del sistema giuridico, legittimando così la sua stessa disobbedienza.

Dall'analisi del conflitto Tap, però, appaiono evidenti i limiti di questo uso del diritto: limiti che sembrano connessi al problema del trattamento del rischio (Corso 2011). La domanda è: cosa può davvero osservare e trattare il diritto davanti al non-sapere del futuro? (Luhmann 2006a). Le decisioni politico-economiche spesso ottengono consenso proprio grazie al non-sapere del futuro, cioè "solo perché le loro conseguenze non possono essere previste. L'intrasparenza del futuro diventa una condizione di possibilità della politica attuale" (Luhmann 2007: 20). Così, il capitalismo scarica sul futuro e sull'ambiente (la natura, i corpi, i sistemi psichici) i suoi principali costi sociali, col vantaggio di nascondere in un aldilà terreno la loro stessa natura di costi. L'intrasparenza del futuro, quindi, "diventa la grande scusa per tutti i misfatti

5 Per approfondire il tema della chiusura del potere è interessante leggere l'analisi di Canetti (1960) sull'addomesticamento delle masse religiose, a partire dai concetti di massa aperta e massa chiusa. 
della nuova società industriale" (Luhmann 1988: 159)6. In questo modo il capitalismo industriale riesce, di fatto, a consentire all'economia e alla politica di sfuggire al controllo del sistema giuridico. Infatti, I'utilizzo politico-economico dell'intrasparenza del futuro rende inerme il diritto rispetto al presente, tempo in cui non si verifica alcun danno, ma si corre solo un rischio/pericolo rispetto a un danno che potrà, eventualmente, solo essere osservato (e giudicato) in futuro. In questo caso il diritto dimostra di essere un sistema strutturato per tollerare la convivenza con il rischio, perché lascia i cittadini nella paura del pericolo e permette all'economia e alla politica di correre rischi in forma (giuridica e) più o meno calcolata, perché limitati solo da valori-soglia standardizzati, cioè non istituiti in base ai differenti contesti ecologico-territoriali in cui vengono costruite le grandi opere (D'Alessandro 2012). "La mia impressione", sostiene Luhmann,

è che aumenti la componente arbitraria nelle regole giuridiche. È necessario definire soglie e scadenze artificiali. Vanno definite unità di misura. È soprattutto necessario fissare un'impostazione sui rischi, per la quale non esistono né certezze di prevedibilità, né una tolleranza del rischio socialmente ammessa. Vengono offerti pigiami per bambini altamente resistenti al fuoco, rispetto ai quali però non è possibile escludere con cerfezza che abbiano un' azione cancerogena (Luhmann 2017: 55)

Pertanto, il conflitto tra diritto e movimenti sociali è, soprattutto, un conflitto sulla distribuzione del rischio, in cui ognuno, anche il diritto come arbitro, si orienta in base al suo non-sapere. II rischio, allora, è un vincolo temporale che consente di trattare il non-sapere, costruendo strutture e fissando aspettative pensate per produrre vantaggi per i decisori che rischiano (l'economia e la politica) e svantaggi possibili per i coinvolti, cioè per chi si trova davanti al pericolo e può soltanto scegliere tra restare inerme o protestare. D'altra parte, le capacità operative del diritto possono essere neutralizzate sia tramite la posticipazione della verifica del rischio/pericolo in un futuro indeterminato, ma anche tramite la procedimentalizzazione del rischio, affinché le operazioni economiche-amministrative possano rientrare nei confini del giuridico. Non è un caso, infatti, che Tap sia riuscita più volte - come ha sostenuto il movimento di protesta - ad eludere "la volontà popolare facendo leva su tecnicismi giuridicolegali": l'esempio più eclatante è l'aggiramento della Legge Seveso, direttiva europea che impone ai propri Stati Membri di identificare i propri stabilimenti industriali a rischio e di seguire delle specifiche procedure autorizzative. La Tap è riusciuta ad eludere i controlli previsti dalla direttiva scorporando la costruzione del suo impianto in due diverse strutture e in questo modo riuscendo a farlo risultare, secondo il Tar del Lazio, "un tipo di impianto non industriale, in cui non si fa né lavorazione né stoccaggio di gas, ma solo trasporto". Anche il Consiglio di Stato ha escluso l'applicabilità della direttiva Seveso e confermato sia la regolarità della Valutazione di Impatto Ambientale sia "I'avvenuto rispetto del principio di leale collaborazione tra Poteri dello Stato nella procedura di superamento del dissenso espresso dalla Regione alla realizzazione dell'opera". In sostanza, nella attuale società del rischio, economia e politica dimostrano di avere gli strumenti per continuare a operare nell'ambito del

\footnotetext{
${ }^{6}$ Traduzione mia.
} 
giuridico, anestetizzando il diritto: tramite l'intrasparenza del futuro oppure seguendo le procedure ed eludendo la legge senza violarla.

Così, dopo che i procedimenti giuridici hanno legittimato i procedimenti amministrativi seguiti da Tap e dal Governo, a fine marzo 2017 sono iniziati i lavori del cantiere. Centinaia di persone, tra cui 15 sindaci, hanno cercato di impedire lo spostamento di 211 ulivi secolari, degli oltre 10.000 di cui è previsto l'espianto. Le forze dell'ordine, in assetto antisommossa, hanno attaccato i manifestanti: il diritto, a cui il fronte del No Tap si era rivolto per cercare la propria legittimità, è diventato improvvisamente un sistema immune alla protesta, un interlocutore sordo, che configura come lecito l'espianto degli ulivi e come illecito la disobbedienza pacifica dei manifestanti. È I'inizio di un conflitto fisico, che però porterà il movimento ad ampliare notevolmente il numero dei suoi sostenitori e anche delle iniziative di confronto e dibattito, a livello locale e nazionale. Ma è anche l'inizio di un disciplinamento giuridico della protesta molto più materiale e di eccezione: il diritto rende manifesto il monopolio della Forza di Legge (Derrida 1992) e della violenza (Benjamin 1920), le misure preventive, la sua maggiore attenzione per i disobbedienti piuttosto che per le forme della disobbedienza, per i corpi piuttosto che per la persona giuridica, il suo identificare il criminale ancor prima del crimine (Foucault 1975). Poco dopo questi scontri, infatti, il Prefetto di Lecce dispone la creazione una zona rossa provvisoria, recintata con mura e filo spinato: 52 manifestanti che si trovano pacificamente nelle adiacenze della zona rossa, vengono aggrediti da poliziotti in assetto antisommossa, arrestati per ore e puniti con pesanti sanzioni amministrative; a due di loro, per il semplice reato di manifestazione non autorizzata e per l'aggravante di accompagnarsi con "soggetti gravati da precedenti penali e/o di polizia per reati dello stesso tenore", sono applicate misure di prevenzione personale previste dal Codice delle leggi antimafia e notificati fogli di via con cui si vieta di "far ritorno nel territorio dei comuni di Lecce e di Melendugno per tre anni". Subito dopo gli arresti e l'emissione dei fogli di via, il Governo intensifica l'atteggiamento repressivo e introduce nella legge di bilancio una misura d'eccezione, cioè un emendamento che trasforma il cantiere in "sito di interesse strategico nazionale", consentendo la militarizzazione e l'arresto (da tre mesi a un anno) per chiunque entri nel cantiere senza autorizzazione. L'emendamento, tuttavia, viene bloccato in quanto lesivo dell'art. 16 della Costituzione.

Così, in un territorio già martoriato dal problema dell'inquinamento, il sistema giuridico ha lasciato che il diritto d'impresa prevalesse sui diritti fondamentáli come il diritto alla salute, costruendo, di fatto, una gerarchia di valori costituzionali e di diritti più o meno rivendicabili. Di conseguenza, il trattamento della protesta è diventato sempre più poliziesco-amministrativo, perché lo Stato ha regolarizzato l'eccezione, trasferendola nelle "periferie sempre meno giuridiche del potere" (Foucault 1975), occupate dalle agenzie della governance neoliberista: i settori del potere giudiziario, gli apparati di polizia, le istituzioni economiche e i mezzi di comunicazione. Si è quindi ottenuta una graduale inclusione giuridica delle operazioni di Tap e un'esclusione del movimento di protesta dai confini del diritto. Pertanto, la disobbedienza civile è stata presto degradata a comune criminalità, nonostante la disobbedienza civile non possa mai essere etichettata come caso particolare di violazione della legge, perché mentre la 
criminalità agisce in modo occulto e per il proprio tornaconto personale, la protesta civile sfida la legge in maniera manifesta e, pur agendo come minoranza, protesta per i diritti di tutta la comunità dei cittadini (Arendt 2017: 23-32). D'altra parte, nonostante la protesta No Tap sia stata stigmatizzata e criminalizzata (tramite arresti, pesanti sanzioni amministrative e fogli di via), non ha mai usato forme di violenza aggressiva, ma al massimo difensiva. E la forma della non-violenza è ciò che distingue una disobbedienza civile da una rivoluzionaria: "coloro che la praticano" - chiarisce Carl Cohen - "accettano l'autorità costituita e la leggittimità generale del sistema giuridico" (Arendt 2017: 32). I- movimento No Tap, infatti, utilizzando tutti i procedimenti messi a disposizione dal -sistema giuridico per opporsi alla realizzazione della grande opera, ha dimostrato pienamente di accettare l'ordine costituito e di inscrivere il suo dissenso esplicito áll'interno del consenso tacito del contratto sociale. Pertanto, se i movimenti dissentono perché altrimenti è come se esprimessero un tacito consenso, allo stesso modo i sistemi politici e giuridici dovrebbero riconoscere che "[l]l dissenso implica consenso ed è il tratto caratteristico di un regime di libertà" (Arendt 2017: 46). Ma questo regime di libertà, evidentemente in modo contro-ideale, sembra comprimersi sempre più.

\section{La funzione ecologica della protesta e della teoria dei sistemi}

Davanti alla forza smisurata dell'economia, della politica e del dirito, viene da chiedersi quale possa essere - per la teoria dei sistemi - l'efficacia e la funzione sociale di un movimento di protesta? Se la società è intesa come universo della comunicazione sociale, la funzione del movimento di protesta non può che essere descritta come prettamente comunicativa. Nella teoria dei sistemi, infatti, le stesse minacce ecologiche non rappresentano dei fatti oggettivi che avvengono nell'ambiente, ma fenomeni esclusivamente interni alla società, che non hanno alcuna rilevanza sociale finché non vengono comunicati:

che diminuiscano le scorte di petrolio, i fiumi diventino caldi, i boschi muoiano, il cielo si offuschi ed i laghi si inquinino. Che tutto ciò avvenga o non avvenga, in quanto stato di fatto solo fisico, chimico o biologico, non produce alcuna risonanza sociale fino a quando non si comunichi su di esso. [...] La società è certo un sistema sensibile, ma operativamente chiuso. Osserva solo attraverso comunicazioni. Non può in altro modo comunicare significativamente e regolare questa comunicazione che attraverso la comunicazione. Si può quindi solo minacciare da sola (Luhmann 1989: 86).

Se le industrie inquinano e le persone muoiono, ma nessuno comunica su questo, è come se ciò non avvenisse: "[L]'ambiente si può rendere percettibile solo attraverso l'irritazione o il disturbo della comunicazione e questa deve quindi reagire a se stessa" (Luhmann 1989: 97). Pertanto, la funzione dei movimenti di protesta può essere solo quella di fornire risonanza a fatti e a cambiamenti dell'ambiente, reali o possibili. La loro funzione è quella di mutare i rapporti di rilevanza fra i valori, di cambiare l'ordine dell'attenzione, di compensare i deficit di riflessione della società moderna, "non facendolo meglio, ma facendolo diversamente" (Luhmann 1991: 163). I movimenti, 
quindi, non hanno la possibilità di cambiare - come si dice - la società, ma solo di selezionare e tematizzare un problema, di produrre attenzione, auspicandone l'inserimento nell'agenda politica. Essi forniscono "contributi all'auto-descrizione della società all'interno della società" (Luhmann 2017: 179), nonostante si comportino come se stessero fuori. In tal modo strutturano un'opposizione centro/periferia: essi sono la periferia della società che protesta contro il suo centro (Luhmann 2017: 192). Tuttavia i loro successi possono consistere solo nell'allarmare la società e verificare come il centro reagisca a questi allarmi, se con indifferenza o meno: "come i cani da guardia i movimenti hanno il forte bisogno di rimettere ordine o almeno di impedire un peggioramento. E come i cani da guardia possono solo abbaiare o mordere" (Luhmann 1991: 164).

Descritta così, la funzione dei movimenti di protesta non sembra avere molte possibilità di incidere sul mutamento sociale. $\mathrm{E}$, infatti, si può facilmente sostenere che i casi in cui riesce ad incidere siano sporadici. Eppure, è grazie ai movimenti di protesta se la società attuale è quella che è, se l'opinione pubblica è cambiata e ha gradualmente accolto le idee rivoluzionarie di alcune minoranze, se i diritti della persona si sono notevolmente ampliati. Pertanto, forse è opportuno tentare di capire chiaramente quale sia il potenziale esplicativo della teoria dei sistemi e come la sua osservazione dei movimenti di protesta possa fornire un contributo ecologico. Qualcuno potrebbe anche obiettare che Luhmann non risponda nel merito alle questioni sociali e filosofico-giuridiche sollevate all'inizio; tuttavia, non si può negare che non lo faccia, ancor più radicalmente, nel metodo. La teoria dei sistemi, infatti, non solo si è interessata alle proteste ecologiste, ma è costruita, essa stessa, come teoria ecologica, perché "la questione dell'ecologia è, in realtà, la questione della teoria sociale, se non la s'intende solo come fatto ambientale, come questione in sé dell'inquinamento, ma come rapporto tra sistema e ambiente" (Luhmann 2017: 61). La prospettiva sistemica, infatti, supera innanzitutto i limiti di ogni approccio antropocentrico, in quanto rinuncia a costruire una teoria sociale a partire dal concetto di vomo, sostituendo la tradizionale distinzione fondativa soggetto/oggetto con quella sistema/ambiente, mettendo così in evidenza come il concetto di 'ambiente' non sia strettamente naturalistico: non solo la società deve fare i conti con il proprio ambiente, ma ogni sistema sociale ha il problema di fare i conti con la complessità di tutto ciò da cui si differenza, ma a cui è interconnesso. In questo modo l'ecologia non è una disciplina che riguarda solo il rapporto tra l'vomo e la natura, ma è una metodologia epistemologica che riguarda in modo trasversale ogni osservazione scientifica che parte, appunto, dalla distinzione tra sistema e ambiente. E poiché ogni sistema è, in base alla prospettiva di osservazione, sia sistema sia ambiente, la stessa distinzione binaria sistema/ambiente non ha più alcun squilibrio simbolico, nessuna connotazione valoriale. La prima conseguenza teorica rilevante per il rapporto tra vomo e natura è che questo rapporto cessa di avere qualsiasi nesso di verticalità o di appropriazione: non c'è più alcuna gerarchia, alcuna supremazia dell'uomo sulla natura. Così, la teoria dei sistemi di Luhmann, come teoria della differenza e della interconnessione, invita a superare non solo l'antropocentrismo giuridico, ma anche la logica appropriativa del diritto, cioè la reificazione della natura 
come bene di possesso dell'vomo. In questo modo essa fornisce alla teoria sociale e giuridica gli strumenti per attuare quella rivoluzione epistemologica tanto invocata dalla nuova dottrina che si occupa di Wild Law e di Earth Jurisprudence ${ }^{7}$. II presupposto ovvio, ma rivoluzionario, dell'approccio sistemico è che qualora si comprometta l'esistenza di un ambiente si comprometterà anche la sopravvivenza del sistema, perché, in una differenza, non può esistere una parte senza l'altra. E così come il diritto e la politica possono compromettere se stessi e la democrazia negando i movimenti di protesta (o viceversa), allo stesso modo la società può compromettere la sua stessa sopravvivenza se rifiuta di assumere, teoricamente ed empiricamente, la sua interconnessione e interdipendenza con il proprio ambiente.

Se la teoria dei sistemi è, quindi, una teoria ecologica, si può facilmente sostenere che anche la funzione della protesta sia una funzione ecologica, al di là se si tratti o meno di una protesta ecologista. La protesta, infatti, può essere descritta come un invito a osservare la periferia, ciò che viene relegato nell'ambiente, l'altra parte occultata dalla latenza. La protesta fa vedere che c'è sempre un ambiente, che sia la natura o che siano i coinvolti, coloro che non decidono ma su cui si ripercuotono le decisioni dei decisori; coloro che possono diventare decisori. La protesta fa vedere che c'è un'altra parte, che l'auto-descrizione della società, quella costruita nel suo centro, dal sistema politico o economico o giuridico, è soltanto una descrizione, senza alcun carattere di necessità. La funzione ecologica della protesta è mostrare la contingenza della realtà, portare il centro nella periferia e la periferia nel centro: far vedere che esistono delle alternative, indicare l'unmarked space, l'altra parte lasciata in ombra. La protesta è ecologia dell'alterità dello sguardo dell'altro: è ecologia della molteplicità.

La questione finale, allora, è se nella società attuale si stia assistendo o meno ad una riduzione delle possibilità di protesta, delle chance dei nuovi movimenti sociali di incidere sul mutamento sociale. Detto in altri termini: benché viviamo nella società della protesta, siamo paradossalmente davanti ad una contrazione delle rivendicazioni collettive dei dirittie Siamo davanti anche a un simultaneo ampliamento delle tecniche per il disciplinamento della protesta o per la sua esclusione dai confini del diritto? $\mathrm{E}$, se così fosse, non sarebbe la stessa democrazia ad auto-escludersi? In

\footnotetext{
7 In campo giuridico, oltre alla Wild Law e alla Earth Jurisprudence, si sono intraprese diverse strade teoriche per costruire una nuova epistemologia ecologica, nel tentativo di rinunciare alla dialettica soggetto/oggetto e di rovesciare lo schema giuridico dell'appartenenza, perché è l'vomo ad appartenere ai sistemi viventi e non il contrario. D'altra parte, l'approccio ecologico consente di far saltare anche altri schemi giuridici come quello del contratto e della responsabilità: perché, da una parte, l'interdipendenza vomo/natura non è negoziabile e, dall'altra, l'aggressione alla sostenibilità della coesistenza uomo/natura non può essere ristorata attraverso forme di compensazione postume. Le strade teorico-giuridiche intraprese per compiere questa rivoluzione epistemologica sono almeno quattro: riconoscere soggettività giuridica a entità biologiche non-umane, come cercano di fare, appunto, la Wild Law e la Earth Jurisprudence; riconoscere e promuovere principi eco-giuridici sul piano dottrinale; sostituire i diritti soggettivi con i doveri di solidarietà (di difesa e protezione), considerando il diritto dell'ambiente come sub-area del diritto amministrativo; infine, in modo più riduttivo, trattare le risorse naturali come beni comuni, mantenendo la distinzione soggetto/oggetto (Monteduro e Tommasi 2015).
} 
effetti, sembrerebbe che - nonostante la società eterarchica - il sistema economico assuma sempre più una funzione primaria e verticistica, favorendo solo quelle acquisizioni evolutive (e quelle operazioni giuridiche) che non contrastino con una costante espansione delle sue possibilità, cioè con la supremazia del denaro - come medium simbolicamente generalizzato (Luhmann e De Giorgi 1991) - sui media degli altri sistemi sociali (Schwartz and Shuva 1992: 205-223). Per questo i movimenti sembrano combattere una lotta impari in cui i sistemi dell'economia, della politica e del diritto non sembrano essere l'uno l'ambiente dell'altro, cioè autonomi e indipendenti, come invece dovrebbero essere in una società moderna. Infatti la politica sembra usare i codici del diritto per legittimare le sue scelte e per rendere l'economia immune ai pericoli del proprio ambiente, intesi come pericoli connessi principalmente alle forme di dissidenza, ormai sempre più sporadiche nella loro effettività pratica.

Se si osserva la realtà attuale è difficile allora imputare a Luhmann un conservatorismo ideologico: è piuttosto l'episteme del presente a restringere gli orizzonti del mutamento sociale. Pertanto, se i movimenti di protesta "come i cani da guardia possono solo abbaiare o mordere" (Luhmann 1991: 164), la sola aspettativa è che la rete in cui il cane è imprigionato possa rompersi o cadere. Oppure che sia il cane, o la natura, a distruggere la rete o a spostare, con essa, i confini giuridici del lecito e dell'illecito, ponendo fine alla criminalizzazione della disobbedienza e all'antropocentrismo appropriativo degli animali umani. Solo così, forse, la società (europea) e il suo diritto potranno liberarsi dalla loro fossilità e accedere allo sguardo dell'altro. Così, ispirati al nuevo costitucionalismo delle Ande (Carducci 2013), immagineranno un nuovo contratto sociale: non soltanto tra persone fisiche, ma tra natura e animali, umani e non. 


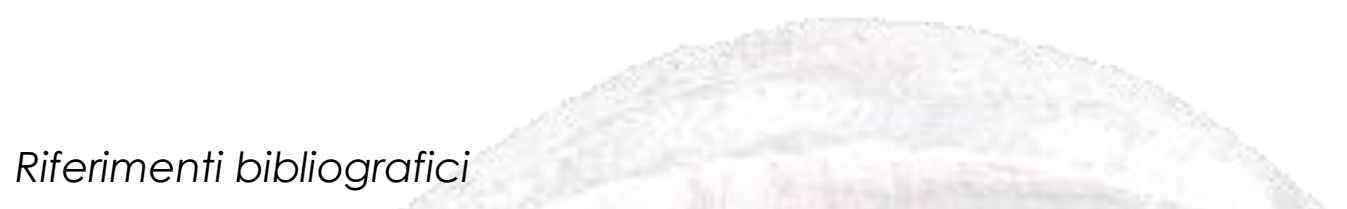

Arendt, H. (2017), Disobbedienza civile, tr. it. Milano: Chiarelettere [Arendt H. (1972), Civil Disobedience, in H. Arendt, Crises of the Republic, (New York: Harvest) 49-102]

Benjamin, W. (1920), "Zur Kritik der Gewalt", in Tiedemann, V.R., and Schweppenhause, H. (eds.) (1999), Walter Benjamin Gesammelte Schriften, (Frankfurt: Suhrkamp) 179-204 Bobbio, L. (2002), 'Discutibile e indiscussa. L'Alta Velocità alla prova della democrazia', 55 II Mulino 124-132

Bobbio, L., Zeppetella, A. et al. (1999), Perché proprio qui? Grandi opere e opposizioni locali (Milano: Angeli)

Bobbio, N. (1992), 'La resistenza all'oppressione, oggi', in Bobbio, N., L'età dei diritti, (Torino: Einaudi) 159-179

Campilongo, C. (2012), Protestas sociales dentro del derecho (Mexico: Ediciones del Hipocampo)

Canetti, E. (2004), Massa e potere, tr. it. Milano: Adelphi [Canetti E. (1960), Mass und Macht (Hamburg: Claassen Verlag)]

Carducci, M. (2013), "La Costituzione come 'ecosistema' nel "nuevo costitucionalismo" delle Ande", in Bagni, S. (a cura di), Dallo Stato del bienestar allo Stato del buenvivir. Innovazione e tradizione nel costituzionalismo latinoamericano (Bologna: Filodiritto) 11-21

Corsi, G. (2003), "La società del rischio", in D'Addario N. et al., Teoria dei sistemi sociali e modernità (Roma: Carocci) 285-296

Corsi, G. (2000), 'Protest and Decision-Making in a Sociefy of Blame', 6 Democracy \& Nature 361-374

Corso, G. (2011), "La valutazione del rischio ambientale", in Rossi, G. (a cura di), Diritto dell'ambiente (Torino: Giappichelli) 172-180

D'Alessandro, F. (2012), Pericolo astratto e limiti-soglia. Le promesse non mantenute del diritto penale (Milano: Giuffrè)

De Giorgi, R. (1998), Scienza del diritto e legittimazione (Lecce: Pensa) 
Della Porta, D. e Diani, M. (2004), Movimenti senza protesta? L'ambientalismo in Italia (Bologna: II Mulino)

Della Porta, D. e Mosca, L. (2002), Globalizzazione e movimenti sociali (Roma: Manifestolibri)

De Nardis, F. (2007), La società in movimento. I movimenti sociali nell'epoca del conflitto generalizzato (Roma: Editori Riuniti)

Derrida, J. (1992), "Force of Law: The 'Mystical Foundation of Authority'" in Cornell D. et al. (eds.), Deconstruction and the Possibility of Justice (London: Routledge) 3-67

De Sanctis, F.M. (1998), "Resistenza (diritto di)", in Enciclopedia del diritto, XXXIX, (Milano: Giuffrè) 987-994

Febbrajo, A. (1975), Funzionalismo strutturale e sociologia del diritto nell'opera di Niklas Luhmann (Milano: Giuffrè Editore).

Foucault, M. (1977), Microfisica del potere. Interventi politici tr. it. Torino: Einaudi

Foucault, M. (1975), Surveiller et punir. Naissance de la prison (Paris: Gallimard)

Jobert, A. (1998), 'L'aménagement en politique-ou ce que la syndrome NIMBY nous dit de l'intérêt général', 42 Politix 67-92

Monteduro, M. e Tommasi, S. (2015), "Paradigmi giuridici di realizzazione del benessere umano in sistemi ecologici ad esistenza indisponibile e ad appartenenza necessaria", in AA.VV., Benessere e regole dei rapporti civili. Lo sviluppo oltre la crisi (Napoli: Edizioni Scientifiche Italiane) 161-202

Lorenz, V. S. (1964), The social movement in France (New Jersey: The Bedminster)

Luhmann, N. (2017), Protesta. Teoria dei sistemi e movimenti sociali, tr. it. Milano: Mimesis [N. Luhmann (1996), Protest. Systemtheorie und soziale Bewegungen, (Frankfurt: Suhrkamp Verlag)]

Luhmann, N. (2010), Potere e complessità sociale, tr. it Milano: II Saggiatore [Luhmann N. (1975), Macht, (Stuttgart: Verlag)]

Luhmann, N. (2006a), Osservazioni sul moderno, tr. it Roma: Armando Editore [Luhmann N (1992), Beobachtungen der Moderne, Opladen: Verlag]

Luhmann, N. (2006b), La sociedad de la sociedad, tr. es. Mexico: Editorial Herder, [Luhmann N. (1997), Die Gesellschaft der Gesellschaft, Frankfurt am Main: Suhrkamp Verlag]

Luhmann, N. (2007), "Metamorfosi dello Stato", in Cevolini, A. (a cura di), Potere e modernità. Stato, Diritto, Costituzione, tr. it. Milano: Angeli, 11-40 [Luhmann N., "Metamorphosen des Staates", in N. Luhmann (1995), Gesellschaftsstruktur und Semantik Studien zur Wissenssoziologie der modernen Gesellschaft, vol. 4, (Frankfurt: Suhrkamp) 101-137]

Luhmann, N. (1996), Sociologia del rischio, tr. it. Milano: Mondadori [Luhmann N. (1991), Soziologie des Risikos, (Berlino: Walter de Gruyter \& Co)] 
Luhmann, N. (1995), Procedimenti giuridici e legittimazione sociale, tr. it. Milano: Giuffrè [Luhmann N. (1969), Legitimation durch Verfahren, Frankfurt: Suhrkamp Verlag]

Luhmann, N. e De Giorgi, R. (1991), Teoria della società (Milano: Angeli)

Luhmann, N. (1989), Comunicazione ecologica. Può la società moderna adattarsi alle minacce ecologiche?, tr. it Milano: Franco Angeli [Luhmann N. (1986) Ökologische Kommunikation. Kann die moderne Gesellschaft sich auf ökologische Gefährdungen einstellen?, Opladen: Verlag]

Luhmann, N. (1988), 'The Third Question: The Creative Use of Paradoxes in Law and Legal History', 15 Journal of Law and Society 153-165

Luhmann, N. (1986), L'alternativa senza alternativa. Il paradosso dei nuovi movimenti sociali, in Luhmann, N. (2017), Protesta. Teoria dei sistemi e movimenti sociali, tr. it. Milano: Mimesis [N. Luhmann (1996), Protest. Systemtheorie und soziale Bewegungen, (Frankfurt: Suhrkamp Verlag)]

Luhmann, N. (1984), Sistemi sociali. Fondamenti di una teoria generale (Bologna: II Mulino).

Luhmann, N., (1979), Potere e complessità sociale, tr. it. Milano: II Saggiatore [Luhmann N. (1975), Macht, Stuttgart: Enke]

Luhmann, N. (1978), Stato di diritto e sistema sociale, tr. it. Napoli: Guida [Luhmann N., Politische Planung, Opladen: Verlag]

Luhmann, N. (1974), "Soziologie als Theorie sozialer Systeme", in Luhmann, N., Soziologische Aufklarung. Aufsatze zur Theorie sozialer System (Opladen: Verlag)

Meyer, D.S. and Tarrow, S. (1998), The Social Movement Society (Lanham: Rowman\&Littlefield)

Noelle-Neumann, E. (2002), The Spiral of Silence. Public Opinion: Our Social Skin (Chicago: University of Chicago Press)

Pennisi, C. (1998), "Teoria e ricerca sociologica sui procedimenti giuridici", in Pennisi, C., Istituzioni e cultura giuridica. I procedimenti come strutture di comunicazione, (Torino: Giappichelli) 145-177

Pieroni, O. (2002), Fuoco, acqua, terra e aria. Lineamenti di una sociologia dell'ambiente (Roma: Carocci)

Schwartz, M. and Shuva, P. (1992), "Resource Mobilization versus the Mobilization of People: why Consensus Movements cannot be instruments of social change", in Morris, A. D. et al. (eds.), Frontiers in social movement theory (New Haven and London: Yale University Press)

Spina, F. (2010), Sociologia dei Nimby. I conflitti di localizzazione tra movimenti e istituzioni (Lecce: Besa)

Thoreau, H.D. (1969), Civil Disobedience: Theory and Practice (New York: Pegasus) 